$V$ is of Type IV (a homology) the group induced by $\Gamma$ on any point of the axis of homology is simply isomorphic with $\Gamma$ (it may not be properly discontinuous, however, in which case $\Gamma$ has no fundamental region). Finally, if $V$ is of Type $V$ (an elation), the induced group on any point of the axis distinct from the center is parabolic and simply isomorphic with $\Gamma$. The method then suffices to determine a fundamental region for every properly discontinuous cyclical group of linear fractional transformations on two complex variables.

It is readily seen, moreover, that the method may be extended to the case where the number of variables is $n$. It will serve also to simplify the problem of determining a fundamental region of any properly discontinuous group of transformations on the points of an $S_{n}$ which leaves a point of $S_{n}$ invariant, provided the group on the $S_{n-k}$ (for some $k=1,2, \cdots, n-1$ ) through this invariant point is simply isomorphic with the given group. The simplification consists in reducing the problem to the determination of a fundamental region for a simply isomorphic group on a smaller number (namely, $k$ ) of variables.

LAWRENCE, KAN., November, 1910 .

\title{
ON THE RELATIVE DISCRIMINANT OF A CERTAIN KUMMER FIELD.
}

BY PROFESSOR JACOB WESTLUND.

(Read before the American Mathematical Society, September 7, 1910.)

Is a paper published in the Transactions of the American Mathematical Society for October, 1910, I determined the fundamental number or discriminant of the algebraic number field $k(\sqrt[p]{m})$, generated by the real $p$ th root of the positive integer $m$. Denoting this discriminant by $d$ and setting $m=a_{1} a_{2}^{2} \ldots a_{p-1}^{p-1}$, where $a_{1} a_{2} \ldots a_{p-1}$ is not divisible by the square of a prime, the following result was obtained :

$$
\begin{aligned}
& d=(-1)^{(p-1) / 2} p^{p-2}\left(a_{1} a_{2} \cdots a_{p-1}\right)^{p-1} \\
& d=(-1)^{(p-1) / 2} p^{p}\left(a_{1} a_{2} \cdots a_{p-1}\right)^{p-1},
\end{aligned}
$$

according as $b^{p-1}-a_{p-1}^{p-1}$ is divisible by $p^{2}$ or not, where $b=$ $a_{1} a_{2}^{2} \ldots a_{p-2}^{p-2}$. 
By adjoining $\rho=e^{2 \pi i p}, p$ being an odd prime, to this field we obtain the Kummer field $k(\sqrt[p]{m}, \rho)$. The object of the present note is to make use of the results mentioned above in determining the relative discriminant $D_{r}$ of this Kummer field with respect to the subfield $k(\sqrt[p]{m})$.

Denoting by $D$ the discriminant of $k(\sqrt[p]{m}, \rho)$, we have *

$$
D=d^{p-1} N\left(D_{r}\right)
$$

Similarly

$$
D=d^{\prime p} N\left(D_{r}^{\prime}\right),
$$

where $d^{\prime}$ is the discriminant of $k(\rho)$ and $D_{r}^{\prime}$ the relative discriminant of $k(\sqrt[p]{m}, \rho)$ with respect to $k(\rho)$. But $d^{\prime}=$ $(-1)^{(p-1) / 2} p^{p-2} \dagger$, hence

$$
D=(-1)^{(p-1) / 2} p^{p(p-2)} N\left(D_{r}^{\prime}\right) .
$$

We have a general method $\$$ for determining the relative discriminant of any Kummer field with respect to $k(\rho)$, but usually the method is of very little practical value. In this particular instance, however, we are able to apply the general method. Two cases arise according as $m$ is divisible by $p$ or not.

\section{I. $m$ not Divisible by $p$.}

Let $c$ be any prime factor of $m$ and $c^{i}(i<p)$ the highest power of $c$ contained in $m$. Then

$$
c=P_{1} P_{2} \ldots P_{e},
$$

where $P_{1}, P_{2}, \ldots, P_{e}$ are prime ideals in $k(\rho)$ of degree $f$, and $p-1=e f$. Now $m$ contains $P_{l}^{i}(l=1,2, \cdots, e)$, and since $i$ is prime to $p$ it follows that $c^{p-1}$ is the highest power of $c$ contained in $D_{r}^{\prime}$. Hence

$$
D_{r}^{\prime}=\left(a_{1} a_{2} \cdots a_{p-1}\right)^{p-1} \lambda^{n}
$$

where $\lambda=(1-\rho)$.

To determine the exponent $n$ we proceed as follows. Let $s$ be the highest exponent $\leqq p$ for which there exists a number $\alpha$

\footnotetext{
*Hilbert, Jahresbericht der Deutschen Mathematiker-Vereinigung, vol. 4 (1894-95), p. 206.

† Hilbert, p. 327.

$\ddagger$ Hilbert, pp. 393-394.
} 
in $k(\rho)$ such that

$$
m \equiv \alpha^{p} \bmod \lambda^{s} .
$$
have

and hence

If $m^{p-1} \equiv 1, \bmod p^{2}$, then $s=p . \quad$ For if $\alpha \equiv m, \bmod \lambda$, we

$$
\alpha^{p} \equiv m^{p}, \bmod \lambda^{p}
$$

$$
\alpha^{p} \equiv m, \bmod \lambda^{p}
$$

since $p=\lambda^{p-1}$. In this case it follows that $D_{r}^{\prime}$ is prime to $p$ and hence $n=0$.

If $m^{p-1} \neq 1, \bmod p^{2}$, then $s=p-1$. For if $s=p$ we should have

and hence

$$
\alpha^{p} \equiv m, \bmod \lambda^{p}
$$

$$
\alpha \equiv m, \bmod \lambda \text {, }
$$

from which follows that

$$
m^{p} \equiv m, \bmod p^{2}
$$

which is contrary to the hypothesis. Hence in this case $\lambda^{2(p-1)}$ is the highest power of $\lambda$ contained in $D_{r}^{\prime}$. Hence $n=2(p-1)$.

But if $m^{p-1} \equiv 1, \bmod p^{2}$, we should have

$$
b^{p} a_{p-1}^{p(p-1)} \equiv b a_{p-1}^{p-1}, \bmod p^{2},
$$

since $m=b a_{p-1}^{p-1}$, and hence

$$
b^{p-1} \equiv a_{p-1}^{p-1}, \bmod p^{2} .
$$

And conversely if $b^{p-1} \equiv a_{p-1}^{p-1}, \bmod p^{2}$, it follows that $m^{p-1} \equiv 1$, $\bmod p^{2}$. Hence

$$
D_{r}^{\prime}=\left(a_{1} a_{2} \cdots a_{p-1}\right)^{p-1}
$$

if $b^{p-1}-a_{p-1}^{p-1}$ is divisible by $p^{2}$, and

$$
D_{r}^{\prime}=\left(a_{1} a_{2} \cdots a_{p-1}\right)^{p-1} \lambda^{2(p-1)}
$$

if $b^{p-1}-a_{p-1}^{p-1}$ is not divisible by $p^{2}$.

\section{II. $m$ Divisible by $p$.}

If $c$ be a prime factor of $m$ different from $p$, it is evident that $c^{p-1}$ is the highest power of $c$ contained in $D_{r}^{\prime}$. And if $p^{i}$ is the highest power of $p$ in $m$, it follows, since $i$ is prime to $p$, 
that $\lambda^{p^{2-1}}$ is the highest power of $\lambda$ contained in $D_{r}^{\prime}$. Hence

$$
D_{r}^{\prime}=c^{p-1} \lambda^{p^{2}-1},
$$

where $c$ is the product of the distinct prime factors of $m$ different from $p$.

From (1), (2), (3), (5), (7), (8), and (9) it then easily follows in both cases that

$$
N\left(D_{r}\right)=p^{p-2} \text {. }
$$

But in the field $k(\sqrt[p]{m})$ we have the following decomposition of $p$ into prime ideal factors, as was proved in the paper mentioned above :

$$
p=P^{p},
$$

if $b^{p-1}-a_{p-1}^{p-1}$ is not divisible by $p^{2}$, and

$$
p=P^{p-1} Q,
$$

if $b^{p-1}-a_{p-1}^{p-1}$ is divisible by $p^{2}$, where $P$ and $Q$ are different prime ideals of the first degree.

In the first case we obtain

$$
D_{r}=P^{p-2} .
$$

In the second case, however, our method does not enable us to determine the exact powers of $P$ and $Q$ which enter into $D_{r}$. We only know that

$$
D_{r}=P^{x} \cdot Q^{y}
$$

where $x+y=p-2$.

PuRdue University, October, 1910.

\section{NOTE ON RECIPROCAL FIGURES IN SPACE.}

BY PROFESSOR PETER FIELD.

Maxwell [Collected Works, page 523. Also see Rankine, Philosophical Magazine for February, 1864] defines figures in three dimensions as reciprocal when they can be so placed that every line in the one is perpendicular to a plane face of the other and every vertex in the one is represented by a closed polyhedron with plane faces in the other.

The simplest case [Maxwell, loc. cit., page 524] is that of 5 